\title{
Gibt es Narzissmus im Kindes- und Jugendalter und wie äußert er sich?
}

\author{
Michael Lipp, Anne Karow
}

\begin{abstract}
Im Kindes- und Jugendalter sind Anlagen von Charaktereigenschaften erkennbar, die sich in der Adoleszenz weiter ausbilden. Narzisstischen Persönlichkeitsmerkmalen kommt hier eine besondere Rolle zu, da sie die Beziehungsfähigkeit mitbestimmen. Es gibt Narzissmus im Kindes- und Jugendalter. Dieser ist jedoch umsichtig zu bewerten, da er stabilisierende Eigenschaften in der Entwicklung einer vielschichtigen, gesunden Persönlichkeit haben kann.
\end{abstract}

\section{Adoleszenz}

Die Adoleszenz birgt als „psychosoziale Pubertät“ eine Vielzahl an Aufgaben im Übergang eines Jugendlichen zu einem jungen Erwachsenen. Die Entwicklungsaufgaben für eine erfolgreich durchlebte Adoleszenz sind uneinheitlich definiert, es scheint aber Einigkeit zu herrschen, dass kulturelle und gesellschaftliche Konventionen wesentlich Einfluss darauf haben (u. a. [1, 2]).

Eine zentrale Aufgabe, unabhängig von den kulturellen Rahmenbedingungen, ist die Entwicklung einer eigenen Identität und Persönlichkeit. Traditionell, in Anlehnung an die bisherigen Diagnosesysteme (ICD-10 und DSM IV bzw. DSM 5), wird auch die Entwicklung von Persönlichkeitsstörungen in dieser Lebensphase verortet. Sofern keine ausreichenden Resilienzfaktoren in der Person selbst oder dem Umfeld des Heranwachsenden vorliegen, kann es im Fall einer gestörten Persönlichkeitsentwicklung, entweder aufgrund frühkindlicher Vulnerabilitätsfaktoren oder auch durch Belastungsfaktoren in der Adoleszenz, zur Ausformung einer Persönlichkeitsstörung kommen [3].

Es gibt Hinweise darauf, dass in der heutigen Gesellschaft die Gefahr einer gestörten Entwicklung in der Adoleszenz erhöht ist. Dies erklären Autoren einerseits durch eine Verlängerung der vulnerablen Phase der Adoleszenz im Vergleich zu vorindustriellen Gesellschaften [4]. Andererseits wird auch eine deutliche Akzeleration der psychosozialen Entwicklung beschrieben. Dies wird beispielhaft mit der Konfrontation mit leicht verfügbaren Informationen in Internet und sozialen Medien, aber auch durch verbesserte Ernährung, Reduktion von Infektionskrankheiten und eine erhöhte Hormonbelastung begründet [5].

\section{Störungsbilder in der Adoleszenz}

In der Adoleszenz manifestiert sich ein Großteil aller psychischen Erkrankungen. Im Alter von 14 Jahren sind be- reits $50 \%$ aller psychischen Erkrankungen nachweisbar, bis zum 24. Lebensjahr manifestieren sich $75 \%$ aller Lebenszeiterkrankungen [6].

Im klinischen Alltag imponieren bei Heranwachsenden häufig sehr dynamische und emotionale Syndrome mit Stimmungsschwankungen, ausgeprägten Gefühlen von Scham, sozialen Ängsten und Befürchtungen, Kränkungen und auch selbstverletzendem Verhalten und Suizidalität. Die Bilder lassen sich nach ICD-10-Kriterien oft am ehesten als Hauptdiagnose einer depressiven Erkrankung oder emotional instabilen Persönlichkeitsstörung zuordnen, wobei psychiatrische Komorbiditäten eher die Regel als die Ausnahme sind (komorbide Suchterkrankung in 50 \% der Fälle, komorbide andere psychische Erkrankung in $66 \%$ der Fälle) [7].

Im Gegensatz zur traditionellen Diagnostik ist es heute allerdings auch üblicher, bei Bedarf Mehrfachdiagnosen zu stellen. Hierdurch soll eine situationsadäquate Beschreibung der Symptomatik erreicht werden. Dabei handelt es sich weniger um eine „Komorbidität“ als um eine „Kosyndromalität“ [8], sodass auch von polysyndromaler Diagnostik gesprochen wird.

Der Auftrag der Adoleszenzpsychiatrie ist einerseits die frühe Erkennung von psychischen Erkrankungen und Bereitstellung einer adäquaten Therapie, um Chronifizierungen zu vermeiden, andererseits die Entstigmatisierung und Vermeidung unnötiger Regressionsförderung und Institutionalisierung bei Patienten, die eher im Rahmen einer Adoleszenzkrise ohne Vorliegen einer manifesten psychischen Erkrankung in das psychiatrische Hilfesystem kommen [9].

\section{Narzissmus}

Der Begriff des Narzissmus wird im allgemeinen Sprachgebrauch in unterschiedlichen Zusammenhängen ver- 
wendet. Selbst in der medizinisch-psychiatrischen Literatur lassen sich kontextabhängig unterschiedliche Schwerpunkte erkennen. Als zentrales narzisstisches Thema wird u. a. das einer erhöhten Kränkbarkeit vor dem Hintergrund einer Diskrepanz zwischen Selbstanspruch und Selbstbild sowie den realen Fähigkeiten und Rückmeldungen aus sozialen Interaktionen beschrieben ([3], S. 390-402).

In der Psychoanalyse hat sich das Konstrukt der „narzisstischen Charakterstörung“ stark gewandelt. Hervorzuheben ist, dass in der Psychoanalyse davon ausgegangen wird, dass aufgrund der einseitigen Libidobesetzung der eigenen Person eine Übertragungsneurose kaum ausgebildet werden kann, weshalb Menschen mit ausgeprägtem Narzissmus schwer behandelbar sind ([3], S. 90-91 und S. 390-402).

\section{Normaler und pathologischer Narzissmus}

Das Konzept eines „normalen und pathologischen Narzissmus" wurde von verschiedenen Autoren entwickelt, um die stabilisierenden Aspekte des Narzissmus einerseits und die destruktiven Seiten andererseits abzubilden $[10,11]$.

Als „normaler Narzissmus“ wird die libidinöse Besetzung des Selbst durch die Integration aggressiver Selbst- und Objektrepräsentanzen in einem kohäsiven Selbstkonzept beschrieben. So hat der „normale Narzissmus“ in der Persönlichkeitsentwicklung stabilisierenden Charakter.

Demgegenüber wird der „pathologische Narzissmus“ als destabilisierend und destruktiv beschrieben. Bei ihm wird ein grandioses Selbst als Abwehr gegen intensive präödipale und ödipale Konflikte im Zusammenhang mit Liebe und Aggression herausgebildet, was zu einer pathologischen Selbststruktur führt [11].

\section{Paradigmenwechsel in der Entwicklung von Persönlichkeitsstörungen}

Der Beginn von Persönlichkeitsstörungen wird in der frühen Kindheit verortet, die Manifestierung in der Adoleszenz angenommen [12]. Bisher lautete daher die Empfehlung, dass die Diagnose einer Persönlichkeitsstörung erst im Erwachsenenalter erfolgen sollte, da sie selten vor dem 16. Lebensjahr voll ausgebildet sei.

In der psychoanalytischen Theorie wird nichtsdestotrotz angenommen, dass Narzissmus in sehr frühen Lebensphasen nicht nur entwickelt wird, sondern auch bereits manifest ist. Hierfür wurden Beobachtungen von Kleinkindern beim Spielen und die Interaktionen mit Spielkameraden und Eltern herangezogen [10]. Die Konsequenzen des Pa- radigmas einer Lebenszeitdiagnose sind ein hohes Risiko der Stigmatisierung der Betroffenen und im ungünstigsten Fall therapeutischer Nihilismus.

Allerdings stützen Langzeituntersuchungen dieses Bild nicht, da auch Persönlichkeitsstörungen in ihrer Symptomausprägung über die Lebenszeit deutlichen Veränderungen unterliegen [13].

Merke

Symptome einer Persönlichkeitsstörungen unterliegen deutlichen Schwankungen. Die Kriterien für eine Persönlichkeitsstörung können zeitweilig erfüllt sein, in anderen Phasen wieder nicht.

Das Gleiche gilt für die psychosozialen Funktionseinschränkungen $[14,15]$. Hier werden Eigenschaften des Temperaments und Persönlichkeitsstile sowie die daraus resultierenden Verhaltensweisen hervorgehoben. Bei entsprechend eingeschränkter Flexibilität in den Verhaltensweisen besteht eine größere Anfälligkeit für Dekompensation im psychosozialen Funktionsniveau. Symptome einer Persönlichkeitsstörung können die Folge sein. Diese können in Krankheitsphasen auch über längere Zeiträume anhalten, aber eben auch wieder remittieren [16].

Im Zuge der Entwicklung eines dimensionalen Ansatzes für Persönlichkeitsstörungen wurde dies berücksichtigt, indem neben der Persönlichkeitsausprägung nach dem Fünf-Faktoren-Modell das Ausmaß der Funktionseinschränkung erhoben wird [3, 17]. Diese Anpassung wurde im Wechsel von dem DSM IV zu DSM 5 berücksichtigt, wobei für den klinischen Teil die bisherige Einteilung des DSM IV beibehalten wurde. Das dimensionale Modell der Persönlichkeitsstörungen findet sich zunächst im Forschungsteil des DSM 5.

\section{- Merke}

Das Paradigma wandelt sich von einer „kranken Persönlichkeit“ hin zu individuell ausgeprägten Persönlichkeitsstrukturen, die mehr oder weniger prädisponierend für eine langfristige Entwicklung einer Persönlichkeitsstörung mit entsprechendem Symptombild sowie sozialen und Alltagsschwierigkeiten sein können [17, 18].

\section{Narzisstische Strukturen in der adoleszentären Entwicklung}

Abhängig von den Entwicklungsphasen kommen unterschiedliche Persönlichkeitsaspekte zur Geltung. Narzisstische Themen, die im Zuge einer adoleszentären Entwicklung stabilisierende Wirkung haben können, sind zum Beispiel die Fähigkeit, Zukunftsperspektiven zu entwickeln, einen Glauben an sich und den eigenen Wert zu haben und Autarkie, also die Fähigkeit, eigene Pläne zu entwickeln 
und umzusetzen. Im Kontext der adoleszentären Entwicklung lassen sich aber auch häufig Fantasien von Großartigkeit, ein großes Bedürfnis nach Bewunderung und nicht selten auch ein (noch) nicht voll ausgebautes Empathievermögen beobachten. Diese Kriterien werden im DSM 5 als „tiefgreifendes Muster“ für die narzisstische Persönlichkeitsstörung zugrunde gelegt [19].

Im Gegensatz dazu fallen häufig schwere Minderwertigkeitsgefühle und Selbstabwertungen auf. Viele Patienten leiden unter mangelhafter Abgrenzungsfähigkeit, was ein ausgeprägtes Empathievermögen voraussetzt. Jugendliche, die mit internalisierenden Störungen, wie Depressionen oder Angststörungen, das psychosoziale Hilfesystem beanspruchen, haben häufig in diesem Bereich Defizite. Hier wäre in Anlehnung an die DSM-Kriterien von einem gering ausgeprägten Narzissmus auszugehen. Diese Beschreibungen können bei Adoleszenten auch parallel oder in kurzer Abfolge bei einem Individuum auftreten, sodass in einem solchen Fall das Zeitkriterium und auch die prinzipielle Definition der Persönlichkeitsstörung als „,tiefgreifendes Muster“ „über einen längeren Zeitraum“ [19] nicht erfüllt sind.

Merke

Die adoleszentäre Entwicklung verläuft nicht linear und in allen Bereichen aufeinander abgestimmt. Heranwachsende mit psychischen Erkrankungen weisen oft Entwicklungsdefizite in mehreren Teilbereichen auf $[2,17]$.

Bei emotional instabilen Syndromen stehen meist dysfunktionale Emotionsregulation und instabile Selbstwert- und Identitätskonstrukte im Mittelpunkt. Die narzisstischen Themen haben Überlappungen mit emotional instabilen Kernbereichen [10].

Hier sei das Selbstbild mit einer bei Adoleszenten meist noch nicht gefestigten Identitätsvorstellung erwähnt, das sich im Fall der Entwicklung einer Persönlichkeitsstörung bei Narzissten zu einem zentralen, tiefgreifenden Thema entwickelt. Die Abspaltung des grandiosen Selbstbilds von Realitätsüberprüfungen ist die notwendige Folge als Abwehr, um auftretende Differenzen aushaltbar zu machen. Die Integration von Bindung und Selbstentfaltung gelingt meist nicht, da das Eingehen von Beziehungen und tatsächliche Nähe schwer aushaltbar sind, solange die Beziehung nicht der Stabilisierung des Selbstwerts dient. Die soziale Kompetenz, die auch eine gewisse Empathiefähigkeit bedingt, weist (Entwicklungs-)Defizite auf.

So gesehen, sind die genannten narzisstischen Aspekte sowohl bei starker als auch bei geringer Ausprägung hinderlich für eine gesunde Entwicklung.
KASUISTIK 1: PATHOLOGISCHER NARZISSMUS ALS HINDERNIS IN DER THERAPIE

Die 19-jährige Frau T. stellte sich mit einem depressiv-suizidalen Syndrom in Begleitung ihrer Mutter in der Zentralen Notaufnahme des Klinikums vor. Stationäre Aufenthalte in der Kinder- und Jugendpsychiatrie vor 4 bzw. 6 Jahren. Sie kenne depressive Episoden und passive lebensmüde Gedanken, habe aktuell aber seit einigen Wochen zunehmende Panikattacken. Vom Hausarzt habe sie Sertralin $50 \mathrm{mg}$ verschrieben bekommen, worunter sich die Angstzustände zugespitzt hätten.

Krisenauslöser sei ein „Sonnenstich“ gewesen: Sie sei im Park gewesen, um sich zu sonnen, und sei dort so bewundert worden, dass sie sich zu lange in der Sonne aufgehalten habe. Ihr sei übel gewesen und sie habe Kopfschmerzen und Herzrasen entwickelt. In den Tagen danach sei die Angst hinzugekommen, in der Öffentlichkeit umzukippen und „die Kontrolle zu verlieren“. Als weiterer Belastungsfaktor zeigte sich, dass Frau T. zwei Monate zuvor von zu Hause ausgezogen war.

Psychopathologisch fiel eine depressive Stimmung mit Grübeln und Gedankenkreisen bei erhaltener Schwingungsfähigkeit auf. Frau T. berichtete von zahlreichen Ängsten, die teilweise sozialen Situationen zugeordnet werden konnten, teilweise aber auch frei flottierten. Weiterhin war ein Verfolgungsund Beobachtungserleben auffällig, sie habe zudem „Menschen im Kopf“, die ihr zuhören würden und ihr Handeln kommentierten. Bei Aufnahme starke Suizidgedanken, von denen sie sich aber klar distanzieren könne. Sie möge sich eigentlich zu sehr, um einen Suizidversuch zu begehen.

Im Stationsgeschehen war das sehr gepflegte und stark geschminkte Erscheinungsbild auffällig. Frau T. verpasste häufig Termine oder kam zu spät, da sie ohne Make-up ihr Zimmer nicht verlassen konnte. Psychotherapeutisch wurde mit viel Validierung gearbeitet, um ein therapeutisches Bündnis zu ermöglichen. In Kontakten wertete sie ihr Gegenüber durch Sprache und Gestik häufig ab und konnte zwischenmenschliche Situationen meist nur über sich selbst überhöhende Bemerkungen gestalten. Dies führte in der Patientengruppe und dem Behandlerteam gegenüber wiederholt zu konflikthaften Situationen. Es gelang im Verlauf, hierüber zu reflektieren und retrospektiv Situationen zu analysieren. So schilderte Frau T. beispielhaft eine Situation, in der sie sich anderen Mitpatientinnen gegenübergesetzt habe, in der Gewissheit, dass sie „besser aussehe“. Sie habe in diesen Momenten Glück verspürt.

In der weiteren Therapie lag das Ziel im Bewusstmachen und Abbau von Bewertungen, was jedoch nur teilweise gelang. Hilfreich waren Elemente aus 
der Acceptance and Commitment Therapy (ACT), wie die Busfahrer-Metapher.

Frau T. äußerte häufig Verfolgungs- und Beobachtungsgefühle in einer Intensität, die teilweise psychotisch anmutete. Im Verlauf ließ sich dies im Rahmen des ausgeprägten Bedürfnisses nach Bewunderung und Aufmerksamkeit einordnen, eine operationalisierte Früherkennungsdiagnostik lieferte keine Hinweise auf ein psychotisches Risikosyndrom. Im Strukturierten Klinischen Interview für DSM IV (SKID II) erfüllte Frau T. die Kriterien für eine narzisstische Persönlichkeitsstörung eindeutig (7/9 Punkte, bei einem Cutoff von 5), für eine paranoide (4/7, Cutoff 4) und Borderline-Persönlichkeitsstörung grenzwertig (5/9, Cutoff 5).

Der Verlauf war von Stimmungseinbrüchen geprägt. Mehrfache Versuche, die Mutter in die Therapie mit einzubeziehen, wurden von der Patientin abgelehnt, Interventionen mit Bezug zur Familie waren nicht möglich. Eine anhaltende Stabilisierung konnte bis zum Ende nicht erreicht werden. Als größte Hürde für nachhaltige Interventionen wurde die Unfähigkeit erlebt, im stationären Setting eine therapeutische Beziehung einzugehen. So wurden jegliche Interventionen allenfalls auf theoretischer Ebene besprochen, eine emotionale Verarbeitung der Inhalte war kaum möglich. Die Entlassung konnte nach sechs Wochen stationärer Krisenintervention nur mit der Aussicht auf eine Wiederaufnahme umgesetzt werden.

KASUISTIK 2: GERING AUSGEPRÄGTE NARZISSTISCHE ZÜGE ALS ENTWICKLUNGSHINDERNIS

Der 18-jährige Herr P. stellte sich auf Zuweisung der Früherkennungsambulanz für Psychische Störungen bei anhaltend depressiver Symptomatik mit sozialem Rückzug und Suizidgedanken geplant zur stationären Behandlung vor. Im Vorfeld war es bereits seit 2 Jahren zu Kontakten in der Kinder- und Jugendpsychiatrie gekommen, aus denen jedoch keine weiterführende Behandlung resultierte. Seit einem Jahr sei Herr P. nicht mehr zur Schule gegangen. Der soziale Rückzug habe sich im Verlauf auf weitere Lebensbereiche erstreckt, sodass er keinerlei Aktivitäten mehr außer Haus unternommen habe. Aufgrund von Ambivalenz gegenüber der Behandlung und Misstrauen erfolgte nach nur einer Nacht auf Station die Entlassung gegen ärztlichen Rat. Zwei Monate später meldete sich Herr P. erneut und es erfolgte eine tagesklinische Aufnahme im Adoleszenzbereich des UKE.

Ein konkreter Krisenauslöser ließ sich nicht eruieren. Herr P. lebte zu Hause mit der Mutter und hatte keinen Schulabschluss. Zum Vater bestand seit Jahren kein Kontakt. Bereits in der Kindheit hatte es zahlreiche Vorstellungen in der Kinderklinik mit unterschiedlichen körperlichen Beschwerden wie Bauchschmerzen gegeben. Herr P. beschrieb, dass sich das Jugendamt aufgrund von Vernachlässigung und Kindeswohlgefährdung bei der Familie eingeschaltet habe, als er 10 Jahre alt war. Seine Mutter habe viele Auflagen erfüllen müssen, um eine Fremdunterbringung zu verhindern.

Psychopathologisch fiel eine etwas manierierte Ausdrucksweise und ein niedergestimmter Affekt mit verminderter emotionaler Schwingungsfähigkeit auf. Herr P. schilderte Insuffizienz- und Schuldgefühle sowie subjektive Wortfindungs- und Konzentrationsstörungen, die in der Außenbetrachtung nicht auffielen. In der Vorgeschichte sei es zu Panikattacken und ritualhaften zwanghaft anmutenden Handlungen gekommen. Er habe mehrfach versucht sich umzubringen, indem er mit geschlossenen Augen über die Straße gegangen sei und einmalig durch Erhängen mit einem Schal. Der Schal sei gerissen. In der Therapie fiel es Herrn P. zunächst schwer, eigene Ziele zu entwickeln. Die Formulierungen waren häufig allgemeingültig und wirkten sozial erwünscht; so wurde als Hauptziel für die Behandlung formuliert, mehr soziale Kontakte zu haben. Das symbiotische Verhältnis zur Mutter fiel auf mit Tendenzen zur Parentifizierung. Versuchen, die Mutter in die Therapie mit einzubeziehen, verweigerte sich Herr P. Er müsse die Mutter schützen, da sie selber auch depressiv sei.

Der Patient profitierte von dem strukturierten Setting und den sozialen Kontakten in der Tagesklinik sowie einem Gruppenangebot zur Depressionsbehandlung. Er hatte in der Patientengruppe die Rolle eines Außenseiters und es gelang ihm nur selten, sich aktiv in der Gruppe zu beteiligen. In der Therapie gelang es, Herrn P. zu aktivieren und die Stimmung leicht zu verbessern. Die Entlassung erfolgte mit der Empfehlung einer Familientherapie, um den Ablösungsprozess von der Mutter zu begleiten und Eigenständigkeit zu fördern.

\section{FAZIT}

Eine gesunde Persönlichkeit setzt sich aus einem breiten Spektrum möglicher Interaktionsstile zusammen, die flexibel eingesetzt werden können. Den Unterschied zwischen einer Persönlichkeitsstörung und einer ausgeprägten, facettenreichen Persönlichkeit macht die Rigidität bzw. die Flexibiltät, in der die unterschiedlichen Aspekte eingesetzt werden können. 
Narzissmus kann sich im Kindes- und Jugendalter sehr destruktiv äußern, indem sich Betroffene mit einem „pathologischen Narzissmus“ aufgrund ihrer mangelhaften Beziehungsfähigkeit und leichten Kränkbarkeit isolieren und in der Erkenntnis, ihrem Selbstbild nicht entsprechen zu können, selbstzerstörerische Tendenzen entwickeln. Auch zu gering ausgebildete narzisstische Persönlichkeitszüge können sich als ungünstig erweisen, wenn der Heranwachsende eigene Bedürfnisse nicht erkennen und keine eigenen Ziele bilden kann.

Ein „normaler Narzissmus“ wirkt hingegen stabilisierend, da er mit einem positiven Selbstbild Sicherheit vermittelt und eine innere Repräsentanz bildet. Diese innere Repräsentanz kann zu einer ausgewogenen Persönlichkeitsentwicklung beitragen. Diese Aspekte sollten in diagnostische und therapeutische Überlegungen einbezogen werden, um die Entwicklungsmöglichkeiten zu nutzen und Chronifizierungen von psychischen Erkrankungen, insbesondere Persönlichkeitsstörungen, entgegenzuwirken.

\section{Interessenkonflikt}

Die Autoren geben an, dass kein Interessenkonflikt besteht.

\section{Autorinnen/Autoren}

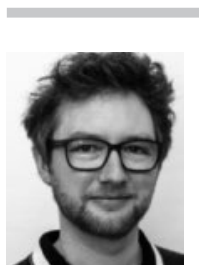

\section{Michael Lipp}

Dr. med. Facharzt für Psychiatrie und Psychotherapie. Klinische Ausbildung in Berlin, Zürich und Hamburg. Funktionsoberarzt auf der Adoleszenzstation an der Universitätsklinik Hamburg-Eppendorf. Schwerpunkte der klinischen Arbeit: Persönlichkeitsstörungen, Früherkennung von psychischen Störungen und Adoleszenzpsychiatrie an der Schnittstelle zwischen der Kinder- und Jugendpsychiatrie und der Erwachsenenmedizin.

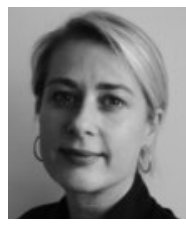

\section{Anne Karow}

Prof. Dr. med. Oberärztin und wissenschaftliche Leiterin des Arbeitsbereichs Adoleszenzpsychiatrie, der Früherkennungsambulanz für Psychische Störungen (FePS) und des Kompetenzzentrums für Integrierte Versorgung an den Kliniken für Kinder- und Jugendpsychiatrie, -psychotherapie und Psychosomatik und der Klinik und Poliklinik für Psychiatrie und Psychotherapie des Universitätsklinikum Hamburg-Eppendorf. Wissenschaftliche Schwerpunkte: Früherkennung und Adoleszenzpsychiatrie, Psychosen und integrierte Versorgung. Leitung des Modellprojekts der sektorenübergreifend-koordinierten, schweregradgestuften, evidenzbasierten Versorgung psychischer Erkrankungen (RECOVER).

\section{Korrespondenzadresse}

\section{Dr. Michael Lipp}

Funktionsoberarzt Adoleszenzstation und -Tagesklinik Klinik für Psychiatrie und Psychotherapie

Zentrum für Psychosoziale Medizin

Universitätsklinikum Hamburg-Eppendorf

Martinistr. 52

20246 Hamburg

m.lipp@uke.de

\section{Literatur}

[1] Erikson EH. Identität und Lebenszyklus. Frankfurt am Main: Suhrkamp; 1973

[2] Havighurst RJ. Developmental Tasks and Education. Chicago: University of Chicago Press; 1948

[3] Fiedler P, Herpertz S. Persönlichkeitsstörungen. Weinheim, Basel: Beltz; 2016

[4] Sawyer SM, Azzopardi PS, Wickremarathne D, Patton GC. The age of adolescence. Lancet Child Adolesc Health 2018; 2: 223-228

[5] Patton GC, Olsson CA, Skirbekk $V$ et al. Adolescence and the next generation. Nature 2018; 554: 458-466

[6] Kessler RC, Berglund P, Demler O et al. Lifetime prevalence and age-of-onset distributions of DSM-IV disorders in the National Comorbidity Survey Replication. Arch Gen Psychiatry 2005; 62: 593-602

[7] Karow A, Lipp M, Schweigert E et al. [Interdisciplinary Inpatient Treatment for Adolescents and Young Adults (16-25 Years) with Mental Illness in Adolescent Psychiatry]. Psychiatr Prax 2018; 45: 248-255

[8] Jager M, Frasch K, Becker T. [Syndromal versus nosological diagnosis]. Nervenarzt 2013; 84: 1081-1082, 1084-1090

[9] Fegert JM, Hauth I, Banaschewski T et al. Eckpunktepapier von DGKJP und DGPPN - Übergang zwischen Jugend- und Erwachsenenalter: Herausforderungen für die Transitionspsychiatrie. DGKJP und DGPPN, Stellungnahmen 2016

[10] Kernberg P. Narzisstische Persönlichkeitsstörungen in der Kindheit. In: Kernberg OF, Hartmann HP, Hrsg. Narzissmus: Grundlagen - Störungsbilder - Therapie. Stuttgart, New York: Schattauer; 2006

[11] Bleiberg E. Normal and pathological narcissism in adolescence. Am J Psychother, 1994; 48: 30-51

[12] Chanen AM, Thompson KN. The Age of Onset of Personality Disorders. In: de Girolamo G, McGorry PD, Sartorius N, eds. Age of Onset of Mental Disorders: Ethiopathogenetic and Treatment Implications. Springer; 2019

[13] Tyrer P. Temporal change: the third dimension of personality disorder. J Pers Disord 2005; 19: 573-580; discussion 594-596

[14] Wright AG, Zalewski M, Hallquist MN et al. Developmental Trajectories of Borderline Personality Disorder Symptoms and Psychosocial Functioning in Adolescence. J Pers Disord 2016; 30: 351-372

[15] Zanarini MC, Frankenburg FR, Hennen J et al. Prediction of the 10-year course of borderline personality disorder. Am J Psychiatry 2006; 163: 827-832

[16] Widiger TA, de Clercq B, de Fruyt F. Childhood antecedents of personality disorder: an alternative perspective. Dev Psychopathol 2009; 21: 771-791 
[17] Tackett JL, Balsis S, Oltmanns TF et al. A unifying perspective on personality pathology across the life span: developmental considerations for the fifth edition of the Diagnostic and Statistical Manual of Mental Disorders. Dev Psychopathol 2009; 21: 687-713

[18] Kaess M, Brunner R, Chanen A. Borderline personality disorder in adolescence. Pediatrics 2014; 134: 782-793

[19] Falkai P, Wittchen HU, Hrsg. Diagnostisches und Statistisches Manual Psychischer Störungen DSM-5. Göttingen: Hogrefe; 2018 